\title{
Experiences with the Execution of Intercultural, Intercontinental Trials - Part I
}

\author{
H.J.M. Barnett
}

\begin{abstract}
Large scale, international clinical trials are formidable challenges, but they are the most effective means of answering important clinical questions in a definitive, generalizable manner. They require adequate funding and must be rigorously conducted. Much can be gleaned from such studies, which address the important research questions and provide answers to related questions. Such trials are enormously rewarding and are worth the expense and effort.
\end{abstract}

\begin{abstract}
RÉSUMÉ: Expérience acquise au cours d'essais cliniques interculturels intercontinentaux. Les essais cliniques internationaux à grande échelle présentent des défis formidables, mais ils constituent le moyen le plus efficace de répondre de manière généralisable et définitive à des questions importantes au point de vue clinique. Ils doivent être financés adéquatement et doivent être menés rigoureusement. Beaucoup d'information peut être recueillie de ces études qui répondent à la question principale et fournissent des réponses à des questions connexes. Ces essais cliniques fournissent des renseignements très utiles et justifient les dépenses et l'effort qu'elles requièrent.
\end{abstract}

Can J Neurol Sci. 2013; 40: 324-329

Reference has been made to several research projects in my memoirs. ${ }^{1}$ During the pursuit of those studies I learned how to conduct, with reasonable success, multi-centre trials. Much has been learned and thousands of patients have consented and committed voluntarily to these trials. Herewith, attempts will be made to summarize the knowledge gained from several large trials in order to outline the essentials involved in the performance of trials, especially those extending beyond national boundaries. There are several absolute essentials of which if any are lacking would be unethical to ask patients to be randomized.

1. There must be a burning clinical problem, important to many in most populations not solvable even by the repeated use of a new drug or technological advance in therapy or diagnosis.

2. When the normal outcomes for an illness vary from spectacular recovery to worsening, only genuine and guarded randomization simultaneously to the old and the putative new therapy have been shown to be capable of producing convincing resolution to the question of treatment value. Anecdotes are of no value and historical controls may mislead.

3. Informed consent requires full understanding by the person asked to submit to random assignment. There are certain irrevocable requirements: a) there is no denying standard treatment to all in the trial. If there is any risk to the innovative therapy it must be fully disclosed to all and understood without equivocation. No additional risk to the innovative therapy is to be tolerated. If the risk of the innovative therapy exceeds what the control group can expect, randomization is not acceptable if the trialist lacks equipoise with the protocol.

4. The annual risks facing patients with the illness under question must be known from pilot observations and with this knowledge, combined with a realistic estimate of the expected benefit of the newer strategy, it will be feasible to calculate the numbers needed in the randomized group and thus sample size can be estimated for a given trial.

5. There must be a control group of equal size, age and gender. This requires from the pilot observations a reasonable estimate of the risk of illness carries to the group receiving standard care.

6. The description of the patients to be randomized must be clearly stated. If there are any sub-groups to be analyzed they must be equally clearly described in the Protocol.

7. All outcome events must be reported to the Central Office of the Trial without delay. These outcomes must be described in prose and by check-offs in the follow-up papers, assuring the reader of consistency. Failure to comply with these requirements is evidence of poor medical conduct and reporting and cannot be tolerated.

Received November 27, 2012. Final Revisions Submitted December 3, 2012 Correspondence to: Henry J.M. Barnett, Suite 701, 305 Balliol Street, Toronto, Ontario, M4S 3H5, Canada. Email: hjmb@bell.net 
8. The outcome details (what can be described as the golddust of every randomized trial) must be sent with all haste to the central office. They will be needed to cope with the "Stopping Rules". The clinical coordinator of the central office must scrutinize each document from every collaborating centre, looking for gaps in obligatory details, or failure to have all reports corrected for English in each centre. Any ambiguities must be reported and discussed immediately with the participating physician in the centre; lack of conformity with the protocol must be recognized and immediately discussed with the investigators in the participating centre. Delays diminish the likelihood of accuracy of recall.

9. The protocol must contain a listing of the patients who are regarded as "Exclusions".

10. The methods planned ahead for the data analysis must be meticulously described in the Protocol. These analyses will form the gold bricks of the trial conclusions.

11. It is mandatory that the protocol, including the Methods of operation of the trial and the methods of analyses be set out and published before the Results paper.

12. The principal investigators, once a Results paper appears, are ethically obliged to respond quickly to any queries about the conduct and the analyses of the trial. The final databank must be regarded as a transparent document available to all once the results have been peer-reviewed and published.

13. If readily understandable, English cannot be anticipated, the centre should not have been recruited; if this need is not carried out in later practice the centre must be reorganized, or be dropped with arrangements for others to care for the patient(s) already randomized. Patients can never be dropped once randomized and, with care, need never be lost.

14. Contributing community physicians will be left in charge of their own patients and regularly be in receipt of progress reports and immediately after examination by a participating stroke neurologist.

15. Stopping Rules must be defined by a biostatistician and in the protocol so that confidential scrutiny of outcome events can betray harm being done or benefit being overlooked.

16. Participants should be rejected who receive consulting fees from industry connected to the subject of the trial

Patients will be examined in routine follow-up at three monthly interval by the participating stroke neurologist or after a presumed outcome event (stroke). Telephone follow-up is not adequate except in unusual and unavoidable circumstances.

Some of the centres may be hemispheres away from the central office. Extreme diligence is required to ensure that each patient in each centre realizes that he or she is of vital importance to the study. The participating centre's coordinator is the lynchpin here and never lets participating subjects feel no longer of importance to the Trial. The stated goal is to have no patients lost to follow-up.

When surgical scars are present, it is part a trial organization to let everyone in the participating centre know that this must be concealed from those evaluating any outcome events. In the North American Symptomatic Carotid Endarterectomy Trial (NASCAT) for example, the Study Manager (Heather Meldrum) and central office Biostatistician (Michael Eliasziw) were totally disciplined about keeping secret the arm of the trial to which each and every outcome patient had been randomly assigned.

Failure to send in timely reports always is a cause for immediate concern. Were they lost or just unable to report? Bill Hass's practice, for example, included some residents living in unsafe parts of the Bronx. He made several prescribed followups as house-calls in these risky places, taking along a burly resident!

The NASCET had two patients whose absence was due to confinement in Federal prisons. ${ }^{2}$ We arranged with the Warden for us to be notified if they were moved by explaining to them the value of their medical data. One patient was a New York reporter imprisoned by Eritrean rebels but he kept in touch and got fully examined when released. Several frail Californian patients decided to return to Mexico. The local and central staff located all of them and filled in their follow-up forms. Expensive, yes but we regarded these studies as demanding perfect data bases.

As our trials expanded, (keeping our goal of 5000 patients in all our combined studies), we insisted on good English translators. In Japan we hired a managing coordinator who spoke perfect Japanese, English and, incidentally, German. If centres could not quickly oblige in this regard, we could not accept the centre. One of our by-pass Japanese surgeons participating in a trial died of a head injury and several of us attended a Buddhist ceremony in his honour. The centre was grateful. We had early learned that the centres were more than just numbers to add to our totals. We did our best to make them our friends and colleagues as well as contributors.

We paid our contributing centres once their documentation was in our hands. We encouraged participating neurologists and surgeons to make visits to our central office and many did! Some wrote manuscripts. If a country flourished numerically we appointed a coordinator to be in charge (as was the case in Japan, Italy and Australia). It removed the potential stigma of "the Canadian study" (to avoid being seen as "somebody else's research").

With growing numbers of centres, one of our participating surgeons was promoted to be the country's supervising surgeon and to be in close touch in his country with all the participating surgeons and report and deal with any irregularities. Dave Sackett shared with Brian Haynes the monitoring of the "best medical care including care of unduly high blood pressure and attempted to ensure "best care" of blood sugar, cholesterol and personal habits.

Once a year we brought together all the participating physicians, surgeons and coordinators to an Annual Bypass or NASCET Meeting to discuss problems, progress and plans for the balance of the trial. These gatherings helped greatly in keeping up enthusiasm. We never discussed results at the Annual Meetings but would discuss unusual outcome reports and encouraged the use of neurological discoveries (e.g. the phenomenon of "near-occlusion"3 and the role of the "carotid stump" 4 in causing symptoms; both of these conditions were first identified in our data-base and participants wrote manuscripts).

There was no template after which to model how we conducted these large trials. Many races, local cultures and egos had to be dealt with. Even on the streets of Belgrade or the 
beaches of Eilat, or the island of Mekong (a possession of Taiwan), we were treated first with politeness then in the end with friendship. For instance, we were given sound advice about dealing with the after-shocks of an earthquake which occurred two days prior to our arrival in Akita. I was not accustomed to seeing the water in the toilet bowl sloshing from side-to-side. I could not "get under the bed" as advised.

I became aware that a multi-centre, multi-racial, multicontinental trial is all-consuming of one's life. The greater the variety of patients and pre-described sub-groups, the more trial results can be generalized. Early on in the Bypass trial we learned that the middle cerebral artery is the artery of cardiac embolism or, in Oriental individuals, of atheroma which much more commonly is a condition in the carotid artery causing stroke in Occidentals. We were taught by Jiro Yonekawa, the PI for Asia of our Bypass Trial the fact that "one size does not fit all". The cardio-thoracic ratio of normal Oriental and certainly Japanese hearts is greater than defined by our original protocol by about $10 \%$. The smaller-statured and slimmer-chested Japanese do not have an enlarged heart identified by this greater cardio-thoracic ratio, and one that would lead to the automatic exclusion of a European or North American candidate.

Following the principles described and with ample help from the late Fraser Mustard, Professors David Sackett, Wayne Taylor, Bryan Haynes and Michael Gent, our University Hospital/ Robarts team was able to complete five separate multi-centre stroke prevention trials. Our system allowed us to claim "almost no patients lost to follow-up" of the approximately 5000 patients randomized in our five trials. A major secret to success was a budget allowing the central office staff to visit the centres regularly; to employ a growing number of research coordinators in the centres, (For phase II of the NASCET Trial the study was paying part or full-time salary equivalents for 128 employees); to hold Annual Meetings of the collaborators and to audit all source data from representative centres chosen at random to verify from hospital chart data already sent in by study forms. If adequate budget allows for an incomplete answer only to the burning question, it should be put aside until such necessary budget items are able to be included. The clinical literature is full of halfanswers and for major issues it is time to await funding appropriate to the impact of the answer on our health-care systems. The best current example to contemplate here is the quarrel over the value of venous stenting in Multiple Sclerosis. Anything short of a well-funded and executed trial is only going to add to the waste and confusion. Individual testimonials often trumpeted in the media and single-centre series reported by enthusiastic major centres pushed cerebral by-pass into undeserved prominence. In the era of evidence-based medicine we are vain enough to believe that our well-funded studies helped to dissipate these ways of approving real advances in therapy.

At weekly central office meetings the outcome data were thoroughly reviewed and, when needed, queries were submitted to the participating centre finalizing clean data for each outcome. At the completion of this tidying-up process, when the outcome events were considered complete, they were submitted to our closely-guarded data-base. One outcome event at a time would be summarized in full detail (excepting the treatment arm assignment) and, when satisfactory to all, would then be submitted to one panel of external reviewers (surgeons and neurologists). Upon return they were ready for final statistical analyses.

This scrupulous process of data-gathering and doublechecking of details was feasible because of the rapid spread of instantaneous electronic communication. The same applied whether the participating centre was in Tokyo, Vancouver, Tel Aviv, Johannesburg or Florence, etc. Multi-continental data could be dealt with as speedily as in single-city studies. For the PI it did not leave time for prolonged holidays PI "escapes" are noted in earlier Memoirs. I did manage to squeeze in three trips to Canada's high Arctic. I admit to learning that there is the compensatory pleasure of satisfaction in completing a challenging project and seeing it through to peer-reviewed publication.

We would not dare claim that we encountered no difficult problems in the execution and conduct of any single trial. Rather there were unexpected problems in all of them that required diplomatic solutions that were soluble in their own particular ways. I will cover them by individual trials. They tended to cover the gamut of human interests and frailties likely to be encountered in clinical research involving hundreds of such rugged individualists as neurologists, vascular and neurosurgeons and radiologists with different types of training and, at times, different prejudices and practices:

I. The Canadian Aspirin Study. ${ }^{5}$ This trial utilized two platelet-inhibiting drugs identified by Fraser Mustard as reducing platelet function. They were tested in a factorial-design trial involving Canadian centres from the Atlantic to the Pacific coasts. Ontario family physicians were not yet widely educated to the concept of double-blind randomization and at a large gathering where the study design was being described and family practitioner cooperation sought, there were questions raised about "using our patients as Guinea-pigs". Many, indeed most of the audience, departed at a call for a show of protest. A later gathering was held in which Dr. Stanley Hagerman, a good community internist and I were able to persuade most of the same and additional local Ontario doctors of the absolutely essential aspect of this method to eliminate biased misrepresentation of results. In the end, in all our trials, Ontario led the way in recruitment. It was not a rare event to hear on the phone from a doctor in southern Ontario: "Hey! I have in front of me a patient who seems to me suitable for your trial. When can you see her?" "What about late this afternoon!"

The description of transient ischemic attack (TIA) as threatening stroke was gradually reaching out into our communities. This was a recognizable secondary benefit of a randomized trial in a medical community. Caring physicians are a necessity to the conduct of organized clinical research.

Halfway through the Canadian Aspirin Study a weak trial was done in which Anturan ${ }^{\circledR}$ was said to be superior in dealing with amaurosis fugax (ocular TIA). This was seized upon by the manufacturer and the drug promoted by advertisements and their detail men as a way to stop stroke in those with warning TIAs. We invited the investigator to talk to our team and we quickly learned that his claim was hollow and his patients' symptoms were multi-causal, many not of vascular origin. They had violated a major principle by not having a clear description of the disorder to be studied. We found a letter in our files in which 
the company had promised us, in writing, that they would not promote this drug until our more rigorous trial was complete. I recall showing this letter to a shaken company representative in the lobby of the Royal York Hotel. He paled and sat down and I extracted a promise that he would report my concerns to his company and make a promise that they would desist from advertising this erroneous claim. They kept their promise. In the end only aspirin proved efficacious and remains the standard for long-term platelet inhibition.

In a much- quoted trial evaluating Clopidogrel against aspirin in three vascular target organs aspirin was quite superior in the group of PVD, inferior in the group entering after a stroke and worse in those with recent myocardial infarction (MI). ${ }^{6}$ Combining outcomes from all three groups, benefit was claimed favoring Clopidogrel (now marketed extravagantly as Plavix ${ }^{\circledR}$ ). This novel change to a combined outcome analysis had never been proclaimed in any preliminary Methods paper. This lack of a Method paper was admitted to me in writing by an officer of the Company. This egregious breech of standard protocol casts a deep shadow over the claimed results. At one point it was common custom to add Plavix to ASA when recurrent TIA occurred. This combined use led to an excess of intracerebral hemorrhages. ${ }^{7}$ Two more points about this flawed trial result: 1) Plavix cost has been as much as sixty times that of aspirin. 2) the stopping of TIA reduces a nuisance. The goal should be to stop stroke of non-cardiac origin!

II. The STA/MCA Anastomosis Trial ${ }^{8}$ (commonly called the Cerebral Bypass Study). Major details of the design of this procedure and its pursuit in a randomized trial has been described earlier in my Memoirs. The trial was conducted in any world-wide centre with experts in the performance of the delicate surgical procedure who were prepared to prove or disprove its efficacy in a randomized and closely supervised trial. The number of active centres was eventually 77, in Asia (Japan, Taiwan. Israel), Europe and North America.

Two separate difficulties erupted in the recruitment of centres: First, several in North America were already convinced of the fact that a by-pass would prevent further ischemic events and that ischemic tissue might be more quickly persuaded to begin functioning again. The term "ischemic penumbra" was generously used to explain this recovery and this catchy phrase made it more difficult to change lucrative practice patterns. Also troubling was the finding by those involved with measuring local cerebral blood flow (both in Texas and in Scandinavia) that they were able to record a decrease after ischemia and then were able, after by-pass, to record an increase towards normal, but unfortunately without substantial evidence of clinical efficacy. The ever-generous and caring Director of the NINDS, Dr. Murray Goldstein, offered to go to prospective but uncommitted centres to help the PI and the Central Office staff to make these distant enthusiasts more aware of the uncertainties hanging over the benefits of the procedure. We made full use of the offer of this recruitment help from a person of such stature in all fields of clinical and surgical trials and went with Murray to make a complete sweep of competent Japanese centres as well as some in the Balkans. Together they proved to be important contributors.

Second, there were misgivings in many places about the distinctively negative results in the total group as well as in every sub-group. One mid-western centre pointed out that in their large group who had the procedure, they could find benefit; so too did the aggregate of Japanese centres. Neither reached statistical significance. When scattergrams were constructed, there were slightly more centres who failed to achieve benefit than those who were more often free of stroke on follow-up. All this simply illustrated the reason for including a wide variety of centres to end up with generalizable conclusions. The doubting Japanese centres finally put together an all-Japanese trial but it again failed. In time the results of our study eventually led to Medicare and Blue Cross deleting cerebral by-pass for ischemia. After they delisted payment for the procedure the controversy subsided.

An NIH-funded trial designed by William Powers, including only patients with positron emission tomography (PET) evidence of defective and hypoxic local metabolism after carotid occlusion, were entered unto a separate NIH-sponsored trial, but after several years it was still proving inconclusive and was abandoned. The "by-pass study" was held up to the Senate Appropriations Committee as evidence supporting the fact that rigorously-pursued clinical studies, albeit costing millions of dollars to conclude in a timely fashion, will spare Health-Care systems and their budgets multiples of millions of dollars and, in a reasonably short time, billions of dollars. Those of us who put the By-pass Study together and gave it nine years of our academic careers were deeply disappointed at the negative results. Friends were lost among surgical colleagues and associations who engaged in a variable measure of harsh criticism of the trial but were not able to find substantial deficits in its design or conduct.

Continuing attempts have been made by enthusiasts to design better by-passes and some for the posterior circulation. They all, so far, carry excess risk and should be abandoned.

III and IV. North American Symptomatic Carotid Endarterectomy Trials. ${ }^{2,9}$ Having completed the Bypass Study, we were asked by NINDS to submit a competing grant to perform a randomized trial of carotid endarterectomy to determine which patients with symptoms arising from an arteriosclerotic internal carotid artery stenosis would benefit. The trial ambitiously set out to test carotid endarterectomy against best medical care. It became apparent early that the trial should be in two simultaneous studies, those with $70 \%$ stenosis or more and those with less. A second goal was to determine the level of operative complications of stroke and death that were compatible with benefit or not. The main results are synopsized earlier in the Memoirs and in the New England Journal of Medicine (NEJM) reports.

The difficulties encountered in this endeavor were manifold. One represented a quarrel between the department of neurosurgery and a senior neurologist in the centre. The latter was peevish and wrote to NIH claiming that the surgeon did not tell the truth about his post-operative complications and this neurologist had reviewed the charts. After many calls to the parties involved, plus a call from the Dean of the Medical school and after looking over the reports we found the problem! The neurologist had overlooked that we requested of all prospective surgeons that we hear about 30-day events of stroke or death. The neurologist had included non-fatal MI, even if diagnosed only by enzyme increase. The Dean knew the surgeon to be a man of exceptional capability and integrity. The centre was a 
good supporter of NASCET. Neuroscience flourishes best when all caring for its disorders work in a harmonious environment!

NASCET trials began with a great deal of resistance from the vascular surgical and neurosurgical community. The President of the American Association of Neurosurgery, representing most of North American neurosurgeons, wrote in an early edition of their official Bulletin expressing the view that this was not a necessary use of National Institutes of Health (NIH) money as "surgeons of experience" already "knew the answer" to a procedure that they had been utilizing for years. Later that year the World Congress of Vascular Surgeons was meeting in New Orleans and with it the business meeting of the American Association of Vascular Surgeons. Two younger members of the latter rose and read out a joint motion asking that the NIH, which had recently announced that they intended to support our grant, reconsider their support. A few members clapped when the motion was read out. The great and revered deBakey rose at once and told the meeting that he hoped that no person would second this motion. He said this was a timely proposal and that even hundreds of anecdotes of stroke prevention did not constitute scientific data proving anything and he strongly supported the $\mathrm{NIH}$ interest in this matter. After a few minutes of tense silence the motion was withdrawn, unseconded; luckily no one clapped. Many American vascular surgeons became participants in our trials of carotid endarterectomy (CE).

Two more crises confronted us in the NASCET trials. First, in response again to slow recruitment and despite the presence of an ongoing very similar trial run from the UK, we decided that we should take in some not yet participating overseas centres; Asiatic centres were not part of our expansion but Australia, Israel and South Africa were to be included. Some of our North American colleagues were slow and reluctant to accept our suggestion and in the end (months later than hoped) these new centres were accepted by our Steering Committee and they made important contributions with acceptable operative risks, well within our demanded range.

Two prominent surgeons, after the trial was published, called up the surgeons at a number of American participating centers. After what their informants disclosed, they managed to elicit an Editorial from the Editors of the New England Journal of Medicine (NEJM) that the confessions of these surgeons had cast a shadow over the trial, as they had not adhered to their written agreements and had not randomized all their eligible patients and that, thereby, the trial had been flawed. By operating on some "ideal candidates" without putting them in the trial, the results might well be distorted. In total these non-randomized patients amounted to fewer than $10 \%$ of those entered. Analyzing the results with and without these patients did not alter any results. As Sackett noted in our collective reply to the Editorial in the NEJM, it is not those who are not in the trial who are as important to the resulting analyses as it is those who are actually randomized who really matter. This matter was the only major criticism brought out by the disappointed, but it delayed for two years the acceptance by the FDA of the results of this multicentre, inter-departmental, inter-racial, multi-continental and multicultural trial. Prior to this, a so-called "Blue Ribbon Committee" of those who disliked the results was constituted. Their audits and machinations have been previously described. The group contained senior neurosurgeons but contained no methodologists, epidemiologists or statisticians. An impartial review was not apparently their goal. Their committee report brought no other problems to light and their final report was in typescript form and ready for publication as our meeting commenced and adjourned. Our complete data-base was made available to them; no committee member gave it a glance nor accepted our offer of full copies. They were not going to alter their critical report by adding new information from us.

The main distress of this quarrel about our competence and therefore our conclusions arose from our protocol-required imaging for the patients of our trial. It came from the persistent demand of one of the NIH reviewers (whose academic life was very dependant on his private ultra-sound laboratory) that we stop insisting on standard angiography and switch the protocol to accept patients with only ultra-sound. Because we had carefully and repeatedly found a $30 \%$ variance between the two methods and had so reported (ultrasound over-reading the degree of stenosis by $30 \%$ )" repeatedly and stubbornly" (the Monitoring Committee's words) we refused to change our protocol and make this switch.

Up until now we had encountered Monitoring Committees who made efforts to help us succeed. This serious confrontation with commerce changed this. We were constantly plagued by our need to keep our recruitment goals. At the end of year five we were still not quite half-way there. We made heroic efforts to satisfy our investigating centres by good communications and contacts. American centres, under the watchful guidance of their particular insurance schemes, were unable to transfer patients to participating centres or to do protocol-determined tests and timed follow-up. The Steering Committee recommended that we add any willing overseas centres not in the UK trial and that we acquire Israeli, South African and Australian help. One very keen US centre in Marshfield, Wisconsin added as many recruits as did the combination of patients entered from centers in New York, Chicago, Detroit and Los Angeles. It had a Board interested in medical advance and was not governed by an insurance corporation.

When Eliasziw, the NASCET statistics expert, evaluated the overseas centres for surgical skill at the end of the trial, he found equal competence in all new and original centers. Not surprisingly, many of the new had been trained at least in part by other collaborators old and new. All had been asked to provide evidence of their known operative risk-level to our Surgical Committee. Later on only two surgeons were asked to leave the trial for a higher than acceptable complication rate. All were on this side of the Atlantic. Their patients remained in the trial. Once randomized all patients are subject to follow-up for the duration of the trial.

At the end of the NASCET trials we were able to conclude that symptomatic patients with $70 \%$ or greater stenosis require CE. With less than $50 \%$, CE may have done more harm than good and was not indicated. With 50-69 "moderate stenosis", benefit would have been realized but muted and would be less than when severe. Patients with near-occlusion benefit in the same proportion as those with moderate stenosis.

V. Aspirin After Carotid Endarterectomy (CE) ${ }^{10}$. In the early days of NASCET, questions were being raised about the timing and dose of the re-starting of aspirin, our recommended platelet inhibitor after CE. We did not have an evidence-based 
answer but learned that a number of our surgeons never stopped what their patients were taking pre-operatively. Thus we decided to use our team of centres and coordinators to help achieve an answer. Any patient within or outside NASCET slated for CE by one of our surgeons was randomized and for 90 days of followup was to receive by random selection either $81,325,925$ or $1300 \mathrm{mg}$ of aspirin daily. We were to learn who had an ischemic event and it was reviewed by our existing NASCET Outcomes Committee. We would record and verify who had a significant GI or cerebral hemorrhage. Final analysis was between half of the patients, those given one of the two lower doses and the other half who were randomized to either of the higher doses. Complications were non-significantly in favor of the lower doses. There was a non-significant increase of hemorrhage with the higher dose, mainly in the $925 \mathrm{mg}$ sub-group. General dose conclusions cannot be drawn for the population of those taking long-term aspirin to prevent stroke. This 90-day follow-up was not long enough to draw dose conclusions. For the balance of NASCET the average dose utilized in our centers was $325 \mathrm{mg}$ daily of enteric-coated ASA begun the day after the procedure.

\section{SUMMARY}

In concluding these huge but detailed trials, we learned several things and select the following as most relevant for those with similar goals:

1. All participants must be enthusiastic and fully familiar with the process under study.

2. Communications with the Central Office and PI'S must be frequent and readily available every day.

3. Participants must know that no deviations from the protocol can be tolerated.

4. The Budget must be adequate to conduct the trial extensively enough to complete the main recruitment goal and to gather all the data

5. "Preliminary" reports have dubious value and may be misleading.

6. Participants are to be encouraged to use the data-base at Trial's end as a rule

\section{REFERENCES}

1. Barnett HJM. Personal reflections from a front-row seat at the greatest show on earth (life); part II (stroke research commentary). Stroke. 2009;40:e53-65.

2. NASCET Collaborators: Beneficial affect of endarterectomy in symptomatic patients with high-grade carotid stenosis. N Eng J Med. 1991;325:445-53.

3. Fox AJ, Eliasziw M, Rothwell PM, Schmidt MH, Warlow CP, Barnett HJM, for the North American Symptomatic Carotid Endarterectomy Trial (NASCET) and European Carotid Surgery Trial (ECST) Group. Identification, prognosis, and management of patients with carotid artery near occlusion. AJNR Am J Neuroradiol. 2005;26(8):2086-93.

4. Barnett HJM, Peerless SJ, Kaufmann JCE. "Stump" of internal carotid artery - a source for further cerebral embolic ischemia. Stroke. 1978;9(5):448-56.

5. The Canadian Cooperative Stroke Study Group (1978). A randomized trial of aspirin and sulfinpyrazone in threatened stroke. N Engl J Med. 1978;299(2):53-9.

6. CAPRIE Steering Committee. A randomized, blinded trial of clopidogrel versus aspirin in patients at risk of ischaemic events (CAPRIE). Lancet. 1996;348(9038):1329-39.

7. Diener HC, Bogousslavsky J, Brass LM, et al. Aspirin compared with clopidogrel alone after recent ischaemic stroke or transient ischemic attack in high-risk patients (MATCH): randomized, double-blind, placebo-controlled trial. Lancet. 2004;364(9431): 331-7.

8. The EC/IC Bypass Study Group. Failure of extracranial-intracranial arterial bypass to reduce the risk of ischemic stroke. Results of an international randomized trial. N Eng J Med. 1985;313(19): 1191-200.

9. Barnett HJM, Taylor DW, Eliasziw M, et al for the North American Symptomatic Carotid Endarterectomy Trial Collaborators. Benefit of carotid endarterectomy in patients with symptomatic moderate or severe stenosis. N Engl J Med. 1998;339 (20):1415-25.

10. Taylor DW, Barnett HJM, Haynes RB, et al for the ASA and Carotid Endarterectomy (ACE) Trial Collaborators. Low-dose and highdose acetylsalicylic acid for patients undergoing carotid endarterectomy: a randomized controlled trial. Lancet. 1999;353 (9171):2179-84 